\title{
Inbreeding and developmental stability in three-spined sticklebacks (Gasterosteus aculeatus L.)
}

\author{
D Mazzi ${ }^{1,4}$, CR Largiadèr ${ }^{2}$ and TCM Bakker ${ }^{3}$ \\ ${ }^{1}$ Division of Behavioural Ecology, Institute of Zoology, University of Bern, Wohlenstrasse 50a, CH-3032 Hinterkappelen, \\ Switzerland; 'Division of Population Genetics, Institute of Zoology, University of Bern, Baltzerstrasse 6, CH-3012 Bern, \\ Switzerland; ${ }^{3}$ Institute for Evolution and Ecology, University of Bonn, An der Immenburg 1, D-53121 Bonn, Germany
}

Fluctuating asymmetry, small non-directional departures from perfect symmetry in bilateral traits, results from the inability of individuals to buffer development against genetic and environmental perturbations. Fluctuating asymmetry is a widely used measure of developmental stability, and developmental stability has been hypothesised to be inversely related to heterozygosity. We compared male three-spined sticklebacks (Gasterosteus aculeatus L.) that had been inbred for one generation to outbred control males

Keywords: developmental stability; fluctuating asymm spined stickleback

\section{Introduction}

Developmental stability refers to the ability of individuals to withstand disruptive genetic and environmental perturbations experienced during development, so as to produce a predetermined optimum phenotype (Waddington, 1942; Lerner, 1954). Fluctuating asymmetry (FA), defined as subtle random deviations from perfect symmetry in bilateral morphological traits, is one widely used measure of developmental stability (Van Valen, 1962; Palmer and Strobeck, 1986). The underlying theory of FA suggests that environmental and genetic stresses during ontogeny reduce the efficiency of normal developmental processes, thus leading to elevated levels of FA (Clarke, 1992). If the phenotypic values of particular characters affect fitness, individuals capable of reliably realising those optimal phenotypes in spite of genetic and/or environmental disturbances are at a selective advantage, and their developmental stability therefore represents an integral component of individual fitness (Clarke, 1995).

The impairing effects of environmental stresses (eg, temperature, pollutants) on developmental stability have been established in a wide range of organisms (see Møller and Swaddle, 1997 for review). As for the genetic basis, developmental stability is supposed to depend upon both genomic coadaptation and heterozygosity (Clarke, 1993).

Correspondence: D Mazzi

${ }^{4}$ Present address: Department of Animal Ecology, Evolutionary Biology Centre, Uppsala University, Norbyvägen 18d, S-75236 Uppsala, Sweden. E-mail: Dominique.Mazzi@ebc.uu.se

Received 27 November 2001; accepted 7 May 2002 with respect to the asymmetry of a set of bilateral morphometric traits. Inbred fish developed significantly more asymmetric pectoral fins than their outbred counterparts, whereas neither the magnitude of asymmetry for pelvic spines nor for gill covers significantly responded to the treatment. Our results conform to a pattern of heterogeneity amongst traits in their tendency to develop asymmetrically in response to stress.

Heredity (2002) 89, 293-299. doi:10.1038/sj.hdy.6800138
Whereas the significance of genomic coadaptation (ie, the co-ordination among gene loci) has been exhaustively documented (Graham, 1992), the role of heterozygosity is still a matter of controversy (Clarke, 1993). Heterozygosity is generally beneficial to individuals, as indicated by positive correlations between heterozygosity and diverse surrogate measures of individual fitness (Britten, 1996). Developmental stability is thought to depend upon heterozygosity, for comparatively superior metabolic efficiency enables heterozygotes to synthesise a wider range of biochemical products, and thus to adjust development to a broader range of environmental and physiological circumstances (Lerner, 1954).

Examples of natural populations in which a cline of heterozygosity matches that of developmental stability (eg, Kat, 1982; Vrijenhoek and Lerman, 1982) have corroborated a consensus about the generality of the heterozygosity effect on developmental stability. However, in a recent meta-analysis, Vøllestad et al (1998) question the ubiquity of the firmly established inverse relationship between heterozygosity and developmental stability, and point out that the available evidence is at best inconsistent, with a number of studies on a wide array of organisms upholding the hypothesis, others failing to support it (Vøllestad et al, 1998 and references therein).

Manipulation of the mating structure of populations offers a way to circumvent many of the pitfalls inherent in tests for a relationship between heterozygosity and developmental stability, such as the estimation of individual heterozygosity and confounding environmental background noise. Any alteration to the normal breeding pattern of a population is known to result in widespread effects on its genetic constitution and physiological 
efficiency (Falconer, 1981). In normally outbreeding populations, the mating of closely related individuals (ie, inbreeding) has long been recognised to adversely affect the fitness of individuals, a phenomenon known as inbreeding depression (Wright, 1921). In the present study, we evaluated the effects of an experimental reduction of average heterozygosity on developmental stability in three-spined sticklebacks (Gasterosteus aculeatus L.). Inbred males (resulted from brother-sister matings) were compared with outbred control males (obtained by randomly crossing unrelated individuals) with respect to the asymmetry of a set of bilateral metric traits. Full-sib matings, as the most extreme form of inbreeding in bisexual species, are regarded as a source of developmental stress, and are thus expected to lead to elevated levels of FA in inbred lines as compared to outbred lines.

\section{Materials and methods}

The measured fish were second-generation $\left(\mathrm{F}_{2}\right)$ laboratory-reared male descendants of fish from a large and genetically heterogeneous anadromous Dutch population. The parental wild fish were collected during the 1998 spring migration on the island of Texel (The Netherlands) and transported to the facilities of the University of Bern in Hinterkappelen (Switzerland), where they were housed individually in 101 plastic aquaria (33 $\times 18 \times 19 \mathrm{~cm}$, water level $15.5 \mathrm{~cm}$ ) with a gravel layer and filamentous algae under summer conditions (16 L:8 $\mathrm{D}$, water temperature $16-19^{\circ} \mathrm{C}$ ). Neighbouring aquaria were separated by grey opaque partitions to prevent visual interactions.

$\mathrm{F}_{1}$-sibships were obtained by crossing sexually mature wild fish at random. Egg clutches were removed from the male's nest $1 \mathrm{~h}$ after fertilisation and placed in aerated plastic beakers with water from a well at $17^{\circ} \mathrm{C}$ refreshed twice a day. Following hatching, in spring 1998, each fullsib group was evenly distributed between two 101 aquaria held under summer conditions and spatially interspersed in random order. Group densities were regularly reduced and equalised by indiscriminately removing some of the fish with a small net. Fish were fed ad libitum twice daily. From December 1998 to March 1999, conditions were switched to winter (8 L:16 D, water temperature $3-6^{\circ} \mathrm{C}$, food in excess once every 2 days). In spring 1999, a random sample of six fish (three per rearing tank) from each of $20 \mathrm{~F}_{1}$-sibships were individually isolated as described for the parental generation. Unrelated $F_{1}$-fish were paired at random to produce full-sib clutches of outbred offspring, while inbred lines resulted from fullsib matings. All fish contributed offspring to one sibship only. The reproduction period was kept as short as possible to ensure that the offspring were a cohort of similar ages. $\mathrm{F}_{2}$-fish were raised following the standardised procedure outlined above. In December 1999, 10 fish per tank (ie, 20 per sibship) were transferred to winter conditions. In March 2000, four males from each of 17 inbred and 18 outbred sibships (two per tank) were randomly selected and isolated individually under summer conditions. All males became reproductively active within a few days of isolation. Within 1 month, between June and July 2000, all fish were killed by decapitation and dissected, beginning with the oldest ones. The treatments are unbiased with respect to age at dissection (median:
416 days, range: 343-436 days for inbred fish, median: 416 days, range: 386-436 days for outbred fish, Wilcoxon rank sum test, $n_{\text {inbred }}=66, n_{\text {outbred }}=71, Z=-0.89, P=0.37$ ).

A condition factor was calculated as $100 \times$ mass (g)/standard length $(\mathrm{cm})^{2.72}$, whereby the exponent in the formula is the slope of the regression of $\log _{10}$ (mass) on $\log _{10}$ (length) (Bolger and Connolly, 1989). Body length and mass were recorded shortly before killing the fish.

For all fish, left and right pectoral fin size, pelvic spine length and gill cover weight were assessed. All traits were measured twice by the same person, the first side to be measured being alternated between successive item pairs, in order to estimate the contribution of measurement error to the variation in asymmetry data (Palmer and Strobeck, 1986). For analyses, we computed the average value of the two measurements, thereby halving the measurement error variance (David et al, 1999). The investigator was blind with respect to the fish genetic make-up at the time measurements were taken. All measurements of damaged or deformed traits were omitted from the analyses, resulting in unequal sample sizes.

Pectoral fins were cut at the basis and dyed in a saturated potassium permanganate $\left(\mathrm{KMnO}_{4}\right)$ solution during 30 sec. Dyed fins were carefully blotted dry on soft tissue paper and spread to a maximum on a glass microscope slide. An image of the fins taken under a binocular microscope at $6.4 \times$ magnification was transferred to an Apple Macintosh computer using a black and white CCD camera, and imported into the public domain image processing and analysis program NIH Image (US National Institute of Health: http://rsb.info.nih.gov/nih-image/) together with a reference label of known dimensions used for calibration. The length of the fin rays was determined by drawing a segmented line all along their centre, from the tip to a straight line set at the fin basis, to the nearest $0.01 \mathrm{~mm}$ (Figure 1a). The outermost two rays were not considered, as bending often precludes precise identification of their extremities. We did not remount the fins between successive measurements to avoid damage, thus we implicitly assume that the preparation itself did not introduce a significant bias. From the sum of the lengths of the eight (exceptionally nine) inner rays we computed an average fin ray length, further used for analyses. The mean length of the inner rays is a good predictor of fin surface, as indicated by the close association between the two variables in a sample of wild parental fish for which both ray length and fin area (as the number of pixels within the outline of the maximally spread fin) were quantified $\left(r^{2}=0.77, n=20, F=\right.$ 61.04 , d.f. $=1,18, P<0.001$ ).

The pelvic girdle with the attached spines was removed from the body, and the spines were separated from the girdle. Pairs of spines were given in a $2 \mathrm{ml}$ Eppendorf-tube with little water and put in a heat chamber at $80^{\circ} \mathrm{C}$ for $1 \mathrm{~h}$, to facilitate subsequent removal of skin residues. Spines were blotted dry on tissue paper and laid on a glass microscope slide with their back facing upwards. Enlarged $(16 \times)$ images of the spines were imported into NIH Image (see above). Spine length was determined as the straight line connecting the lowest point of the articulation to the spine tip, to the nearest $0.01 \mathrm{~mm}$ (Figure 1b). The image analysis program was recalibrated prior to every pair of measurements.

The gill covers were torn off at their joint and stored in pairs in a $2 \mathrm{ml}$ Eppendorf-tube at $-20^{\circ} \mathrm{C}$ until further 
a

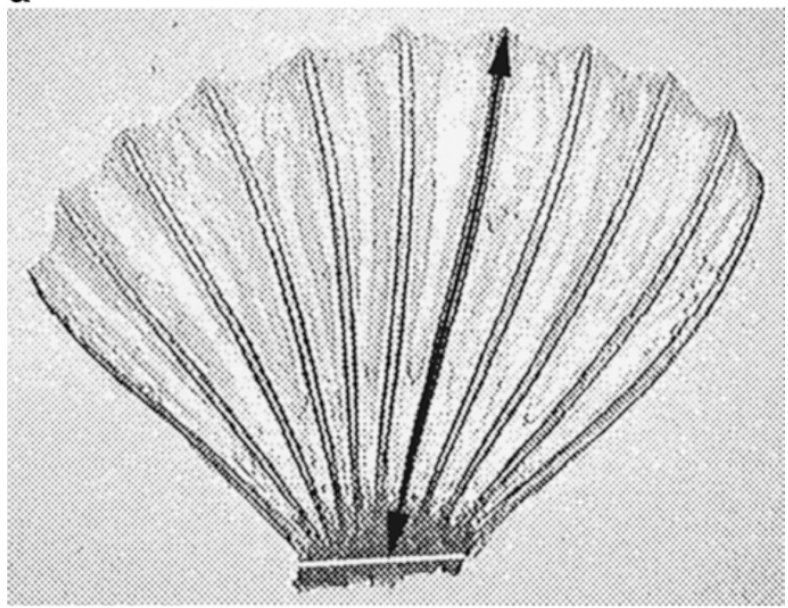

b

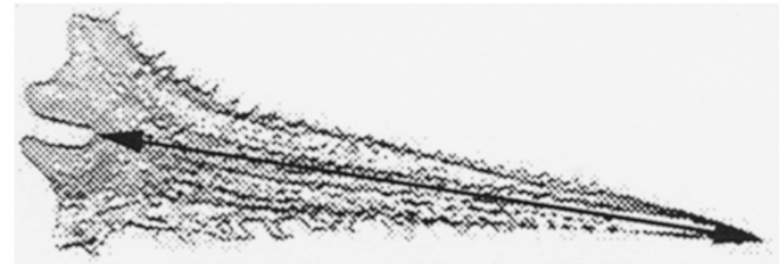

Figure 1 Measurement of traits: (a) Fin ray length was given by a segmented line connecting the ray tip to a line set at the fin basis. For analyses, the average length of the eight (exceptionally nine) inner rays was used. (b) Spine length was given by the straight line connecting the lowest point of the articulation to the spine tip.

processing. Later, they were put for $2 \mathrm{~h}$ in a heat chamber at $80^{\circ} \mathrm{C}$ to soften coarse skin, flesh and cartilage remnants, and were meticulously scraped clean with the aid of forceps and a toothbrush soaked with absolute ethanol. Gill covers were dried for $2 \mathrm{~h}$ in a heat chamber at $80^{\circ} \mathrm{C}$. Their weight is stable afterwards (B Kobler, personal communication). Dried gill covers were weighed on an analytic electric balance (Sartorius Supermicro S4, $\pm 10^{-6} \mathrm{~g}$ ) to the nearest microgram.

Asymmetry analysis and measurement error appraisal were based on an approach modified from Leamy (1984) and recommended by Palmer and Strobeck (1986). A two-way, mixed-model analysis of variance (ANOVA) was performed on the two within-subject repeated measurements for each trait, considering the factors side (fixed), individual (random) and their interaction. The ANOVA measures population $\mathrm{FA}$ as the variance between left and right sides. Moreover, it provides information about the magnitude of measurement error as a proportion of the total variance for each character, and allows the detection of directional asymmetry (ie, the trait value on one side is consistently larger than its counterpart). Kurtosis was calculated to test for the presence of platykurtosis, indicating antisymmetry (ie, asymmetry is the norm but the side with the larger trait value varies). We tested for a relationship between the magnitude of FA and character size by regressing absolute trait asymmetry on trait size. Inbred and outbred lines were tested separately.

The effect of the fish genetic background on the response variables was analysed using mixed-model, nested ANOVA models, with treatment (inbred or outbred) as fixed factor and sib group (nested within treatment) as random factor.

We used the index FA9a, ie $1-r$ where $r$ is the Pearson's correlation coefficient between left and right trait values to compare FA across traits (Windig and Nylin, 2000).

Two-tailed $P$-values are given throughout. Analyses were performed using JMP IN (v. 4.0.3, SAS Institute, 2000) statistical package.

\section{Results}

The asymmetry was fluctuating in pectoral fins and pelvic spines, as indicated by the lack of side effects in the ANOVAs (Table 1). Significance of the side term for gill covers indicated directionality for this trait, with right trait values being consistently larger than left (Table 1). Kurtosis was positive for all traits, providing no evidence of antisymmetry. The distribution of asymmetry for the considered traits is illustrated in Figure 2. The asymmetry far exceeded measurement error in all traits, as demonstrated by the highly significant 'Side' $\times$ 'Individual' interaction terms of the mixed-model ANOVAs (Table 1). The variation in FA for inbred and outbred lines is summarised in Table 2.

Pectoral fin asymmetry and size were significantly negatively associated for inbred fish $\left(r^{2}=0.11, n=56, F\right.$ $=6.62$, d.f. $=1,54, P=0.013$; Figure 3 ), not significantly so for outbred ones $\left(r^{2}=0.0005, n=60, F=0.03\right.$, d.f. $=$ $1,58, P=0.87)$. Save one outlier, the lower bound of the plot of asymmetry against size was flat (Figure 3), indicating that the increase in asymmetry as trait size declines reflects increased FA rather than antisymmetry (Rowe et al, 1997). The magnitude of measurement error did not depend upon trait size $(P=0.9)$, thus the observed relationship was not likely due to small traits being more severely inflated by measurement error than larger traits (Yezerinac et al, 1992). Gill cover asymmetry was not significantly associated with gill cover weight, neither for inbred $\left(r^{2}=0.03, n=63, F=2.18\right.$, d.f. $=1,61$, $P=0.15)$ nor for outbred fish $\left(r^{2}=0.02, n=69, F=1.52\right.$, d.f. $=1,67, P=0.22$ ). Pelvic spine asymmetry showed no obvious relationship to spine length, either for inbred or outbred fish $\left(r^{2}=0.005, n=50, F=0.28\right.$, d.f. $=1,48, P=$ 0.60 and $r^{2}=0.01, n=59, F=0.76$, d.f. $=1,57, P=0.39$, respectively). In the absence of evidence for positive sizedependence, neither trait was scaled for size, ie the unsigned difference between left and right trait values (|L-R $\mid$ ) was used for analyses.

Pectoral fins responded to the treatment in the expected direction, ie inbred fish developed significantly more asymmetric pectoral fins than outbred fish (Table 3). In contrast, neither the magnitude of asymmetry for pelvic spines, nor for gill covers differed significantly with respect to the fish genetic background (Table 3). These conclusions hold true after Bonferroni-adjustment to account for multiple related tests. The asymmetry of any given trait did not predict the asymmetry of the others ( $P>0.1$ for all pairwise correlations).

Significant family effects were detected for the size of all measured traits, but no evidence for treatment effects [pectoral fins: $F_{33,81}=2.94, P<0.0001$ for the effect of sib group (nested within treatment), $F_{1,81}=0.58, P=0.45$ for the effect of treatment; pelvic spines: $F_{31,76}=5.14, P<$ 0.0001 for the effect of sib group (nested within 
Table 1 Type of asymmetry and reliability of asymmetry measurements

\begin{tabular}{|c|c|c|c|c|c|c|c|c|c|c|c|c|}
\hline & \multicolumn{4}{|c|}{ Individual } & \multicolumn{4}{|c|}{ Side } & \multicolumn{4}{|c|}{ Individual $\times$ Side } \\
\hline & MS & d.f. & $F$ & $P$ & MS & d.f. & $F$ & $P$ & MS & d.f. & $F$ & $P$ \\
\hline \multicolumn{13}{|l|}{ Inbred } \\
\hline Pectoral fins & 0.829 & 56,113 & 61.178 & $<0.0001$ & 0.002 & 1,113 & 0.128 & 0.722 & 0.014 & 56,113 & 26.675 & $<0.0001$ \\
\hline Pelvic spines & 1.691 & 49,100 & 35.510 & $<0.0001$ & 0.017 & 1,100 & 0.363 & 0.550 & 0.048 & 49,100 & 9.279 & $<0.0001$ \\
\hline Gill covers & 0.448 & 62,126 & 151.523 & $<0.0001$ & 0.017 & 1,126 & 5.897 & 0.018 & 0.003 & 62,126 & 306.084 & $<0.0001$ \\
\hline \multicolumn{13}{|l|}{ Outbred } \\
\hline Pectoral fins & 0.991 & 59,120 & 112.861 & $<0.0001$ & 0.002 & 1,120 & 0.196 & 0.660 & 0.009 & 59,120 & 11.677 & $<0.0001$ \\
\hline Pelvic spines & 1.877 & 58,118 & 23.537 & $<0.0001$ & 0.017 & 1,118 & 0.219 & 0.642 & 0.080 & 58,118 & 1792.636 & $<0.0001$ \\
\hline Gill covers & 0.448 & 68,138 & 189.736 & $<0.0001$ & 0.019 & 1,138 & 8.039 & 0.006 & 0.002 & 68,138 & 32.227 & $<0.0001$ \\
\hline
\end{tabular}

Significance of the 'Individual' term indicates that the differences between individuals are larger than the measurement error. Significance of the 'Side' term indicates the presence of directional asymmetry. Significance of the 'Individual $\times$ Side' term indicates that the asymmetry is larger than the measurement error.

treatment), $F_{1,76}=0.08, P=0.77$ for the effect of treatment; gill covers: $F_{33,97}=4.13, P<0.0001$ for the effect of sib group (nested within treatment), $F_{1,97}=0.04, P=0.84$ for the effect of treatment]. Fish with larger fins also had longer spines and heavier gill covers $(P<0.0001$ for all pairwise correlations), as expected given the positive allometry of the considered characters with body size (all $P<0.001$ ).

Body condition differed significantly among sibships, however, not between inbred and outbred lines $\left[F_{33,102}=\right.$ 2.29, $P=0.0008$ for the effect of sib group (nested within treatment), $F_{1,102}=0.49, P=0.49$ for the effect of treatment]. Fish with relatively high physical condition did not develop larger or more symmetric traits, as compared to fish in poorer condition $(P>0.08$ for all pairwise correlations).

\section{Discussion}

Developmental stability, as measured in terms of pectoral fin size asymmetry, was disrupted upon inbreeding in three-spined sticklebacks, consistent with the hypothesis that decreased morphological stability results from lowered heterozygosity (either per se or from an enhanced expression of deleterious recessive alleles) (Lerner, 1954). However, male sticklebacks that had been inbred for one generation exhibited significantly higher levels of FA than their outbred control counterparts in only one of three examined morphometric traits. Levels of pectoral fin size asymmetry were significantly elevated in inbred fish, supporting other authors' suggestion that pectoral fins represent a sensitive character for monitoring developmental stability in fish (eg, Moran et al, 1997). In contrast, the asymmetry of neither pelvic spines nor gill covers significantly responded to the treatment, nor did the average size of any trait.

In a recent survey of the available experimental evidence for patterns of FA in relation to stress, Bjorksten $e t$ al (2000) pointed out that the response of FA to stress is trait-, species- and stress-specific, yielding striking within-study inconsistencies. Often, levels of FA increase in only one of several examined traits, in other instances traits vary in their response to different stressors (Bjorksten et al, 2000 and references therein). The specificity of asymmetry has been argued to limit its adequacy to gauge stress, since its indicator potential crucially depends on both the chosen traits to score and the particular stressor investigated (Bjorksten et al, 2000). The typically low concordance for FA across traits within individuals has been imputed to different traits being affected by different stresses, or having different periods of heightened sensitivity to developmental perturbations (Van Valen, 1962; Møller and Swaddle, 1997). However, others have argued that, given the small proportion of variance a trait's FA on average owes to individual differences, no higher among-traits correlations than those commonly reported are to be expected (Gangestad and Thornhill, 1999). Here, asymmetries of the examined characters within the same individual did not detectably predict one another. Sticklebacks thus apparently add to the numerous taxa in which different stressors elicit a reaction on different traits. An increase in levels of pelvic spine asymmetry of juveniles followed a medium-term exposure to acid stress (Mazzi and Bakker, 2001), while in this study, pectoral fins were the most severely impaired trait.

Besides being the primary propulsive organ for routine locomotion and serving manoeuvrability, the pectoral fins are used by males during the parental phase to fan water through the nest (Bell and Foster, 1994). In sticklebacks, a male's parental ability crucially affects reproductive success, as the male alone cares for the offspring for about 2 weeks by defending the eggs against predation and ventilating them by regular beats of the pectoral fins (Wootton, 1976). The dual use of pectoral fins in locomotion and parental care likely makes high functional demands on the size and the shape of the pectoral fins. The size of the pectoral fins is sexually dimorphic in three-spined sticklebacks, with males having larger fins than females during the breeding season (Bakker and Mundwiler, 1999). Pectoral fin size affects paternal quality, as demonstrated by an experimental reduction of fin size leading to extended time required for the young to hatch (Künzler and Bakker, 2000). Bilateral symmetry is likely to raise both fanning efficiency and manoeuvrability, and thus ought to be favoured by natural selection and/or sexual selection through female choice of good fathers. Females relying on a hypothetical indicator of male heterozygosity in their choice of mates may additionally derive indirect benefits by producing a higher proportion of heterozygous, genetically diverse progeny (Brown, 1997). It seems implausible that females 


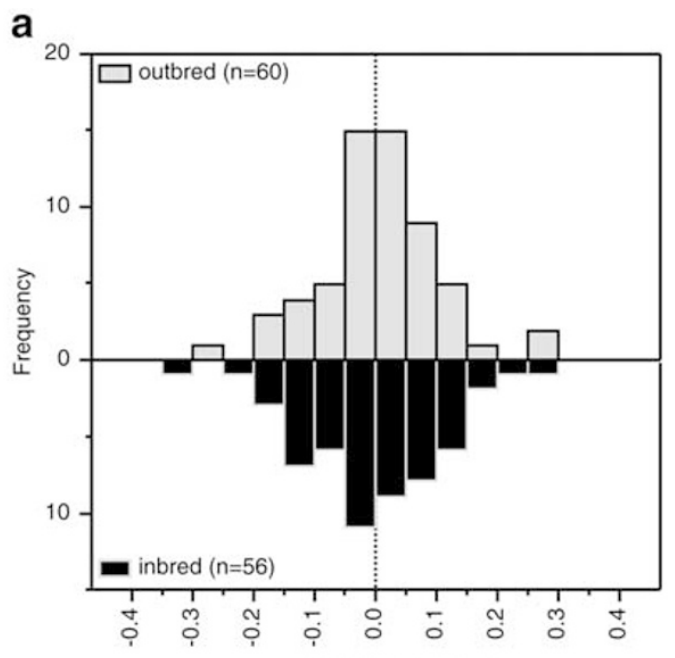

b

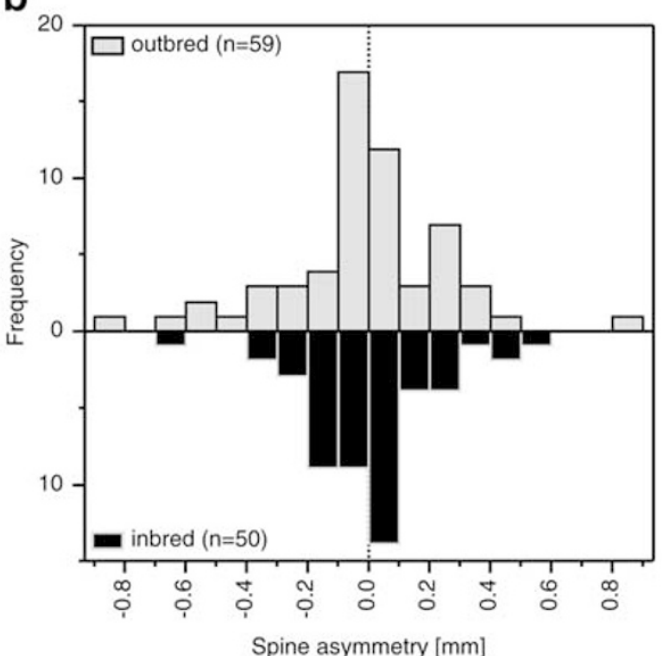

C

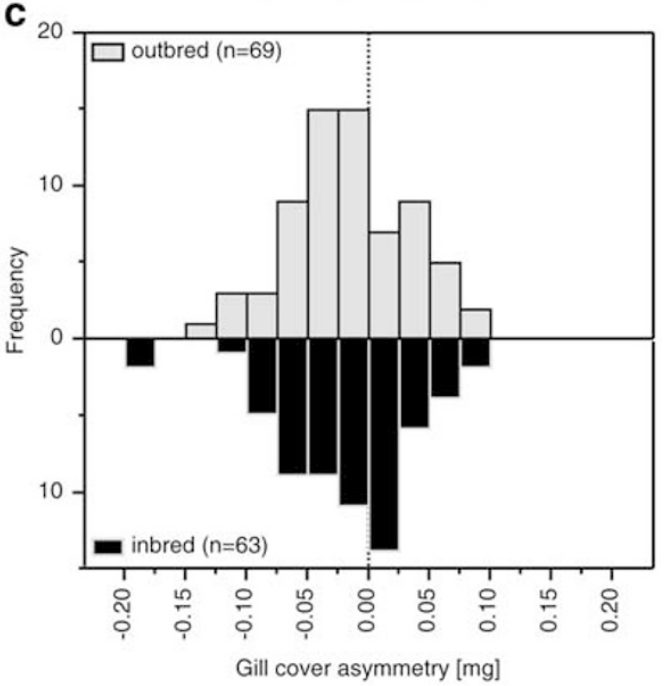

Figure 2 Frequency distribution of the signed difference between the left and right pectoral fin size (a), pelvic spine length (b), and gill cover weight (c) for outbred and inbred fish.
Table 2 Mean ( \pm s.d.) asymmetry of pectoral fins, pelvic spines and gill covers, as the average of replicate line means within each treatment. Unsigned absolute asymmetry is measured as the unsigned difference of left minus right trait value, ie $|\mathrm{L}-\mathrm{R}|$. Signed size-corrected asymmetry is measured as the signed difference of left minus right trait value divided by the average trait value, ie (L$\mathrm{R}) /[(\mathrm{L}+\mathrm{R}) / 2]$. Unsigned size-corrected asymmetry is measured as the unsigned difference of left minus right trait value divided by the average trait value, ie $|\mathrm{L}-\mathrm{R}| /[(\mathrm{L}+\mathrm{R}) / 2]$. Index FA9a, where $r$ is the Pearson's correlation coefficient between left and right trait values, is recommended by Windig and Nylin (2000) for comparing FA across traits. The sample size is 17 inbred and 18 outbred lines for pectoral fins and gill covers, 15 inbred and 18 outbred lines for pelvic spines

Trait Inbred Outbred

Unsigned absolute asymmetry

$\begin{array}{lll}\text { Pectoral fins [mm] } & 0.10 \pm 0.04 & 0.07 \pm 0.04 \\ \text { Pelvic spines [mm] } & 0.17 \pm 0.06 & 0.21 \pm 0.14 \\ \text { Gill covers [mg] } & 0.04 \pm 0.02 & 0.04 \pm 0.02\end{array}$

Signed size-corrected asymmetry [\%]

$\begin{array}{lrr}\text { Pectoral fins } & -0.05 \pm 0.94 & 0.11 \pm 0.75 \\ \text { Pelvic spines } & 0.18 \pm 1.34 & -0.08 \pm 2.71 \\ \text { Gill covers } & -1.15 \pm 1.53 & -1.12 \pm 1.59\end{array}$

Unsigned size-corrected asymmetry [\%]
Pectoral fins
$1.31 \pm 0.56$
Pelvic spines
$2.46 \pm 0.93$
Gill covers
$2.74 \pm 1.51$
$0.87 \pm 0.46$
$3.07 \pm 1.96$
$2.80 \pm 1.30$

Index FA9a: 1-r [ \pm 95\% confidence limit]

Pectoral fins

0.031

0.017

Pelvic spines

$[0.018 ; 0.052]$

$[0.010 ; 0.028$

0.073

Gill covers

[0.030; 0.090]

0.013

$[0.044 ; 0.120]$

0.010

[0.008; 0.021]

[0.006; 0.016]

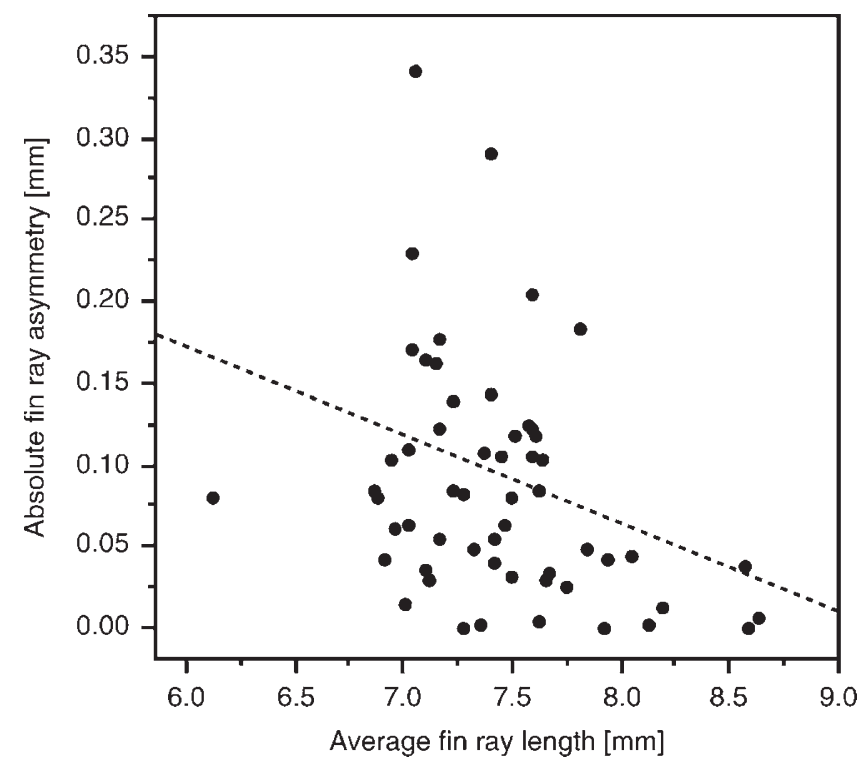

Figure 3 Relationship between pectoral fin size and asymmetry for inbred fish. Fin asymmetry is given as the unsigned difference of left minus right fin size. 
Table 3 Effects of treatment (inbred or outbred, fixed effect) and sib group (nested within treatment, random effect) on the absolute asymmetry of pectoral fins, pelvic spines and gill covers

\begin{tabular}{lrccc}
\hline Source of variation & d.f. & MS & $F$ & $P$ \\
\hline Pectoral fins & & & & \\
$\quad$ Treatment & 1 & $8.1 \times 10^{-4}$ & 6.426 & $0.016^{*}$ \\
$\quad$ Sib group [Treatment] & 33 & $1.3 \times 10^{-4}$ & 0.930 & 0.582 \\
$\quad$ Error & 81 & $1.4 \times 10^{-4}$ & - & - \\
Pelvic spines & & & & \\
$\quad$ Treatment & 1 & 0.057 & 1.450 & 0.237 \\
$\quad$ Sib group [Treatment] & 31 & 0.039 & 1.454 & 0.096 \\
$\quad$ Error & 76 & 0.027 & - & - \\
Gill covers & & & & \\
$\quad$ Treatment & 1 & $8.4 \times 10^{-6}$ & 0.006 & 0.940 \\
$\quad$ Sib group [Treatment] & 33 & 0.001 & 1.470 & 0.076 \\
$\quad$ Error & 97 & 0.001 & - & - \\
\hline
\end{tabular}

${ }^{*} P<0.05$ (after Bonferroni correction)

assess differences in the size of the transparent, quite inconspicuous pectorals directly. However, reliable information about fin morphology might be obtained through correlated traits such as the courtship dance, which involves repeated abrupt changes of speed and direction and thus may be influenced by pectoral fin asymmetry. Moreover, during a courtship sequence, males regularly visit their nest to perform behaviours pertinent to parental care, including fanning (Sevenster, 1961; Bakker and Mundwiler, 1999). Males thereby presumably deceive females by pretending to have eggs in their nest, as females preferentially spawn in nests that already contain eggs (Goldschmidt et al, 1993), but may alternatively advertise parental ability and/or individual heterozygosity to choosy females.

Sexual traits are generally supposed to be particularly sensitive to the effects of stress, owing to a recent history of directional selection and condition-dependent expression (Møller and Pomiankowski, 1993). Comparing the asymmetry of different traits is not straightforward (Windig and Nylin, 2000). Nevertheless, as measured in terms of the index FA9a (Windig and Nylin, 2000), gill covers exhibited the lowest asymmetry, followed by pectoral fins and pelvic spines (Table 2), and indeed the latter two traits probably are a target of sexual selection (Künzler and Bakker, 2000; D Mazzi, R Künzler and TCM Bakker, unpublished data). However, pectoral fins serve locomotion and paternal care (see above), while pelvic spines are part of an armour complex protecting against vertebrate predation (Bell and Foster, 1994), and natural selection should oppose FA in structures with a mechanical function to be performed effectively. Hence, the characters' functionality should not be neglected when examining FA-size relations or comparing asymmetry levels across traits (cf. Hunt and Simmons, 1998). For gill covers, the asymmetry was directional rather than fluctuating, with right gill covers being consistently heavier than left. The mean of the asymmetry distribution significantly deviating from zero is not likely a consequence of the measurer's handedness, as weight measurements are immune to such bias, and probably neither reflects a functional advantage. Whether or not directional asymmetry (DA) is suited as a predictor of developmental stability is a debated issue (eg, Kraak, 1997). Whereas FA has been firmly established as a measure of developmental precision, owing to its presumed exclusive environmental basis, the use of DA has been advised against because of its assumed genetic component (Leary and Allendorf, 1989). However, even heritability estimates of individual FA are sometimes different from zero (Møller and Thornhill, 1997 and references therein, but see also Leamy, 1997; Pomiankowski, 1997; Whitlock and Fowler, 1997), indicating an additive genetic component to developmental stability, which has obviously not affected the popularity of FA as a monitor of stress. On the other hand, although a heritable basis has sometimes been detected (Leamy et al, 1997), the average genetic variance of DA accounted for can be very low, and the observed phenotypic variation overwhelmingly environmental in its origin (Leamy et al, 2000), thus entitling the use of DA as a predictor of developmental stability. Our measure of asymmetry (unsigned difference between left and right) does not take DA into account, but our conclusions do not change if DA in gill covers is corrected for by subtracting the mean signed asymmetry from the signed asymmetry values.

As with any negative result, we cannot conclusively rule out the failure of detecting existing effects of inbreeding on the asymmetry of pelvic spines and gill covers. For example, our results are conservative with respect to selective mortality eliminating the most extreme phenotypes at young age (Floate and Fox, 2000). Samples being measured for FA consist of a selection of individuals that survived exposure to stress, rather than of the totality of individuals exposed to stress. The more developmentally unstable individuals in the population are most likely to exhibit elevated levels of FA in response to stress, yet least likely to survive its effects. With increasing levels of stress, the proportion of more robust individuals displaying low levels of FA increases at the expense of more susceptible individuals displaying high levels of FA, and average FA declines. Moreover, the average reduction of heterozygosity realised after a single generation of full-sib matings (corresponding to an inbreeding coefficient of $F=0.25$ ) may not be severe enough a stress to evoke a considerable reduction of developmental stability in terms of FA in some traits.

In summary, we report decreased developmental stability in terms of pectoral fin size asymmetry, but not in terms of pelvic spines or gill covers asymmetry, following an experimental manipulation of the genetic constitution of three-spined sticklebacks. By comparing inbred with outbred lines, we circumvented the ambiguity of heterozygosity estimates, while through standardised rearing conditions we eliminated the confounding influence of potentially covarying environmental factors, two pitfalls that have seriously hindered the interpretation of a number of previous studies of developmental stability in relation to heterozygosity, particularly for vertebrates.

\section{Acknowledgements}

We thank Bernd Kobler, Reto Künzler and Marc Zbinden for discussion, Alex Kohler and Judith Zbinden for assistance, Heinz Richner for providing facilities, Jürg Zettel for allowing the use of the analytic balance, and the referees for helpful comments on the manuscript. The study was funded by the Swiss National Science Foundation. 


\section{References}

Bakker TCM, Mundwiler B (1999). Pectoral fin size in a fish species with paternal care: a condition-dependent sexual trait revealing infection status. Freshwat Biol 41: 543-551.

Bell MA, Foster SA (1994). Introduction to the evolutionary biology of the threespine stickleback. In: Bell MA, Foster SA (eds) The Evolutionary Biology of the Threespine Stickleback, Oxford University Press: Oxford. pp 1-27.

Bjorksten TA, Fowler K, Pomiankowski A (2000). What does sexual trait FA tell us about stress? Trends Ecol Evol 15: 163-166.

Bolger T, Connolly PL (1989). The selection of suitable indices for the measurement and analysis of fish condition. J Fish Biol 34: 171-182.

Britten HB (1996). Meta-analyses of the association between multilocus heterozygosity and fitness. Evolution 50: 2158-2164.

Brown JL (1997). A theory of mate choice based on heterozygosity. Behav Ecol 8: 60-65.

Clarke GM (1992). Fluctuating asymmetry: a technique for measuring developmental stress of genetic and environmental origin. Acta Zool Fenn 191: 31-35.

Clarke GM (1993). The genetic basis of developmental stability. I. Relationships between stability, heterozygosity and genomic coadaptation. Genetica 89: 15-23.

Clarke GM (1995). Relationships between developmental stability and fitness: application for conservation biology. Cons Biol 9: 18-24.

David P, Hingle A, Fowler K, Pomiankowski A (1999). Measurement bias and fluctuating asymmetry estimates. Anim Behav 57: 251-253.

Falconer DS (1981). Introduction to Quantitative Genetics. Longman: London.

Floate KD, Fox AS (2000). Flies under stress: a test of fluctuating asymmetry as a biomonitor of environmental quality. Ecol Appl 10: 1541-1550.

Gangestad SW, Thornhill R (1999). Individual differences in developmental precision and fluctuating asymmetry: a model and its implications. J evol Biol 12: 402-416.

Goldschmidt T, Bakker TCM, Feuth-de Bruijn E (1993). Selective copying in mate choice of female sticklebacks. Anim Behav 45: 541-547.

Graham JH (1992). Genomic coadaptation and developmental stability in hybrid zones. Acta Zool Fenn 191: 121-131.

Hunt J, Simmons LW (1998). Patterns of fluctuating asymmetry in beetle horns: no evidence for reliable signaling. Behav Ecol 9: 465-470.

JMP (2000). Version 4. SAS Institute Inc., Cary, NC.

Kat PW (1982). The relationship between heterozygosity for enzyme loci and developmental homeostasis in peripheral populations of aquatic bivalves (Unionidae). Am Nat 119: 824-832.

Kraak SBM (1997). Fluctuating around directional asymmetry? Trends Ecol Evol 12: 230.

Künzler R, Bakker TCM (2000). Pectoral fins and paternal quality in sticklebacks. Proc R Soc Lond B 267: 999-1004.

Leamy L (1984). Morphometric studies in inbred and hybrid house mice. V. Directional and fluctuating asymmetry. Am Nat 123: 579-593.
Leamy L (1997). Is developmental stability heritable? J evol Biol 10: $21-29$.

Leamy LJ, Routman EJ, Cheverud JM (1997). A search for quantitative trait loci affecting asymmetry of mandibular characters in mice. Evolution 51: 957-969.

Leamy LJ, Pomp D, Eisen EJ, Cheverud JM (2000). Quantitative trait loci for directional but not fluctuating asymmetry of mandible characters in mice. Genet Res Camb 76: 27-40.

Leary RF, Allendorf FW (1989). Fluctuating asymmetry as an indicator of stress: implications for conservation biology. Trends Ecol Evol 4: 214-217.

Lerner IM (1954). Genetic Homeostasis. Wiley: New York.

Mazzi D, Bakker TCM (2001). Acid stress increases pelvic spine asymmetry in juvenile three-spined sticklebacks. J Fish Biol 59: 582-592.

Møller AP, Pomiankowski A (1993). Fluctuating asymmetry and sexual selection. Genetica 89: 267-279.

Møller AP, Swaddle JP (1997). Asymmetry, Developmental Stability, and Evolution. Oxford University Press: Oxford.

Møller AP, Thornhill R (1997). A meta-analysis of the heritability of developmental stability. J evol Biol 10: 1-16.

Moran P, Izquierdo JI, Pendas AM, Garcia-Vazquez E (1997). Fluctuating asymmetry and isozyme variation in Atlantic salmon: relation to age of wild and hatchery fish. Trans Am Fish Soc 126: 194-199.

Palmer AR, Strobeck C (1986). Fluctuating asymmetry: measurement, analysis, patterns. Ann Rev Ecol Syst 17: 391-421.

Pomiankowski A (1997). Genetic variation in fluctuating asymmetry. J evol Biol 10: 51-55.

Rowe L, Repasky RR, Palmer AR (1997). Size-dependent asymmetry: fluctuating asymmetry versus antisymmetry and its relevance to condition dependent signaling. Evolution 51: 1401-1408.

Sevenster P (1961). A causal analysis of a displacement activity (fanning in Gasterosteus aculeatus L.). Behaviour Suppl 9: 1-170.

Van Valen L (1962). A study of fluctuating asymmetry. Evolution 16: $125-142$

Vøllestad LA, Hindar K, Møller AP (1998). A meta-analysis of fluctuating asymmetry in relation to heterozygosity. Heredity 83: 206-218.

Vrijenhoek RC, Lerman S (1982). Heterozygosity and developmental stability under sexual and asexual breeding systems. Evolution 36: 768-776.

Waddington $\mathrm{CH}$ (1942). Canalization of development and the inheritance of acquired characters. Nature 150: 563-565.

Whitlock MC, Fowler K (1997). The instability of studies of instability. J evol Biol 10: 63-67.

Windig JJ, Nylin S (2000). How to compare fluctuating asymmetry of different traits. J evol Biol 13: 29-37.

Wootton RJ (1976). The Biology of the Sticklebacks. Academic Press: London.

Wright S (1921). Systems of mating. II. The effects of inbreeding on the genetic composition of a population. Genetics 6: 124143.

Yezerinac SM, Lougheed SC, Handford P (1992). Measurement error and morphometric studies: statistical power and observer experience. Syst Biol 41: 471-482. 